\title{
Classical Spin Nematic Transition in $\mathrm{LiGa}_{0.95} \mathrm{In}_{0.05} \mathrm{Cr}_{4} \mathrm{O}_{8}$
}

\author{
R. Wawrzyńczak,,${ }^{1, *}$ Y. Tanaka, ${ }^{2, \dagger}$ M. Yoshida, ${ }^{2}$ Y. Okamoto, ${ }^{3}$ P. Manuel, ${ }^{4}$ N. Casati, ${ }^{5}$ \\ Z. Hiroi, ${ }^{2}$ M. Takigawa, ${ }^{2}$ and G. J. Nilsen ${ }^{4, \sharp}$ \\ ${ }_{1}^{1}$ Institut Laue-Langevin, 6 rue Jules Horowitz, 38042 Grenoble, France \\ ${ }^{2}$ Institute for Solid State Physics, University of Tokyo, 5-1-5 Kashiwanoha, Kashiwa, Chiba 277-8581, Japan \\ ${ }^{3}$ Department of Applied Physics, Nagoya University, Furo-cho, Chikusa-ku, Nagoya 464-8603, Japan \\ ${ }^{4}$ ISIS Neutron and Muon Source, Science and Technology Facilities Council, Didcot OX11 OQX, United Kingdom \\ ${ }^{5}$ Swiss Light Source, Paul Scherrer Institute, 5232 Villigen PSI, Switzerland
}

(Received 16 February 2017; published 22 August 2017)

\begin{abstract}
We present the results of a combined ${ }^{7} \mathrm{Li}$-NMR and diffraction study on $\mathrm{LiGa}_{0.95} \mathrm{In}_{0.05} \mathrm{Cr}_{4} \mathrm{O}_{8}$, a member of the $\mathrm{LiGa}_{1-x} \mathrm{In}_{x} \mathrm{Cr}_{4} \mathrm{O}_{8}$ "breathing" pyrochlore family. Via specific heat and NMR measurements, we find that the complex sequence of first-order transitions observed for $\mathrm{LiGaCr}_{4} \mathrm{O}_{8}$ is replaced by a single secondorder transition at $T_{f}=11 \mathrm{~K}$. Neutron and x-ray diffraction rule out both structural symmetry lowering and magnetic long-range order as the origin of this transition. Instead, reverse Monte Carlo fitting of the magnetic diffuse scattering indicates that the low-temperature phase may be described as a collinear spin nematic state, characterized by a quadrupolar order parameter. This state also shows signs of short-range order between collinear spin arrangements on tetrahedra, revealed by mapping the reverse Monte Carlo spin configurations onto a three-state color model.
\end{abstract}

DOI: 10.1103/PhysRevLett.119.087201

Spinel materials, $A B_{2} X_{4}$, host a variety of interesting magnetic phenomena, including spin-orbital liquid states $\left(\mathrm{FeSc}_{2} \mathrm{~S}_{4}\right)$ [1], Skyrmion lattices $\left(\mathrm{GaV}_{4} \mathrm{~S}_{8}\right)$ [2], and magnetostructural transitions [3-6]. Many of these originate from the $B$ site, which forms a frustrated pyrochlore lattice of corner-sharing tetrahedra. When the large spin degeneracy caused by the frustration is combined with strong magnetoelastic coupling, a generic feature of spinels, several possible magnetostructurally ordered and disordered states arise. For example, in the chromate spinel oxides, $A \mathrm{Cr}_{2} \mathrm{O}_{4}$, collinear, coplanar, and helical magnetic structures (and their accompanying structural distortions) may all be realized by varying the cation on the $A$ site $[3,4,6,7]$. Although the low-temperature behavior of the chromates is complex, it is well captured by the bilinearbiquadratic model (BBM) [8,9], the Hamiltonian of which is $\mathcal{H}=J \sum_{i, j} \vec{S}_{i} \cdot \vec{S}_{j}+b \sum_{i, j}\left(\vec{S}_{i} \cdot \vec{S}_{j}\right)^{2}+\mathcal{P}$, where $\vec{S}_{i, j}$ are classical Heisenberg spins and $J$ is the nearest-neighbor exchange. The second and third terms represent respectively the magnetoelastic coupling, which assumes a biquadratic form if only local distortions are considered, and perturbative terms such as further neighbor couplings. These act on the degenerate manifold of states $\sum_{i \in \operatorname{tet}} \vec{S}_{i}=0$ (where the sum is over tetrahedra) generated by the Heisenberg term, in turn selecting either collinear $(b<0)$ or coplanar $(b>0)$ configurations [8], and then breaking the remaining degeneracy and establishing magnetic order $(\mathcal{P} \neq 0)[10,11]$.

Two recent additions to the chromate spinel family are the so-called "breathing" pyrochlore materials, $A A^{\prime} \mathrm{Cr}_{4} \mathrm{O}_{8}$, where $A=\mathrm{Li}^{+}$and $A^{\prime}=\mathrm{Ga}^{3+}, \mathrm{In}^{3+}$ [12]. Here, the alternation of the $A$ and $A^{\prime}$ cations on the $A$ site leads to a corresponding alternation in tetrahedron sizes and, hence, magnetic exchange constants, $J$ and $J^{\prime}$, on the pyrochlore lattice. This alternation is quantified by the breathing factor $B_{f}=J^{\prime} / J$, which is $\sim 0.6$ for $A^{\prime}=\mathrm{Ga}^{3+}$ and $\sim 0.1$ for $A^{\prime}=\operatorname{In}^{3+} . B_{f} \neq 1$ notwithstanding, the phenomenology of the breathing pyrochlores at low temperature is similar to their undistorted cousins. In both $A^{\prime}=\mathrm{Ga}^{3+}(x=0)$ and $A^{\prime}=\mathrm{In}^{3+}(x=1)$, a sequence of two transitions (cf. lightly doped $\mathrm{MgCr}_{2} \mathrm{O}_{4}$ [13]) lead to structural and magnetic phase separation (cf. $\mathrm{ZnCr}_{2} \mathrm{O}_{4}$ [14]), while the excitation spectra show gapped "molecular" modes in the ordered states (cf. $\mathrm{MgCr}_{2} \mathrm{O}_{4}$ [15]). In $x=0$, the upper magnetostructural transition at $T_{u} \sim 20 \mathrm{~K}$ results in phase separation into cubic paramagnetic and tetragonal collinear phases [16,17]; the cubic phase then undergoes another first-order transition into a second tetragonal phase at $T_{l}=13.8 \mathrm{~K}$, the structure of which is unknown [19]. Interestingly, this transition is preceded by a divergence in the ${ }^{7} \mathrm{Li}$-NMR $1 / T_{1}$, implying proximity to a tricritical point [20].

Studies of the solid solutions $\mathrm{LiGa}_{1-x} \mathrm{In}_{x} \mathrm{Cr}_{4} \mathrm{O}_{8}$ indicate that $T_{l}$ is rapidly suppressed when $x$ is increased or decreased from $x=0$ or 1 . Starting from $x=0$, sharp peaks in the magnetic susceptibility and specific heat persist until $x \sim 0.1$, beyond which they are replaced by features characteristic of a spin glass [21]. This side of the phase diagram resembles those of both $\mathrm{Zn}_{1-x} \mathrm{Cd}_{x} \mathrm{Cr}_{2} \mathrm{O}_{4}$ [22] and Monte Carlo (MC) simulations of the BBM with $b<0$ disorder and further neighbor interactions $\mathcal{P}=J_{n n n} \sum_{i, j} \vec{S}_{i} \cdot \vec{S}_{j}$ [23].

In this Letter, we show that the low-temperature behavior of the $x=0.05$ composition, apparently well inside the 
magnetically ordered regime of the phase diagram, differs drastically from $x=0$. Instead of two first-order transitions and phase separation, we observe a single second-order transition using ${ }^{7} \mathrm{Li}-\mathrm{NMR}$ and specific heat. Remarkably, this transition corresponds neither to magnetic long-range order nor a structural transition. Rather, the magnetic diffuse neutron scattering implies that it shares features with both the nematic transition predicted for the BBM on the pyrochlore lattice and the partial ordering transition expected for the pyrochlore antiferromagnet with further neighbor interactions [11]. Furthermore, the transition is shown to coincide with spin freezing, drawing parallels to other frustrated materials, like $\mathrm{Y}_{2} \mathrm{Mo}_{2} \mathrm{O}_{7}$ [24,25].

The powder samples of $\mathrm{LiGa}_{0.95} \mathrm{In}_{0.05} \mathrm{Cr}_{4} \mathrm{O}_{8}$ were prepared via a solid-state reaction [12,21], using ${ }^{7} \mathrm{Li}$ enriched starting materials to reduce neutron absorption. The ${ }^{7} \mathrm{Li}-\mathrm{NMR}$ measurements were carried out in a magnetic field of $2 \mathrm{~T}$, and NMR spectra were obtained by Fourier transforming the spin-echo signal. At low temperatures, the spectra were constructed by summing the Fourier transformed spin-echo signals measured at equally spaced frequencies. The nuclear spin-lattice relaxation rate $1 / T_{1}$ was determined by the inversion-recovery method at the spectral peak [26]. The specific heat was measured using the relaxation method on a Quantum Design Physical Property Measurement System (PPMS).

The samples were further studied by powder synchrotron $\mathrm{x}$-ray diffraction (SXRD) on the MS-X04SA beamline at Paul Sherrer Institute (PSI) (Switzerland), and powder neutron time-of-flight diffraction (ND) on the WISH instrument at ISIS (UK). For the former, patterns were measured in the temperature range 6-20 K using a photon energy of $22 \mathrm{keV}$ $(\lambda=0.564 \AA$ ), while for the latter, the temperature range was 1.5-30 K. Structural refinements were carried out on both data sets (Fig. 2) using the FULLPROF software [27]. The
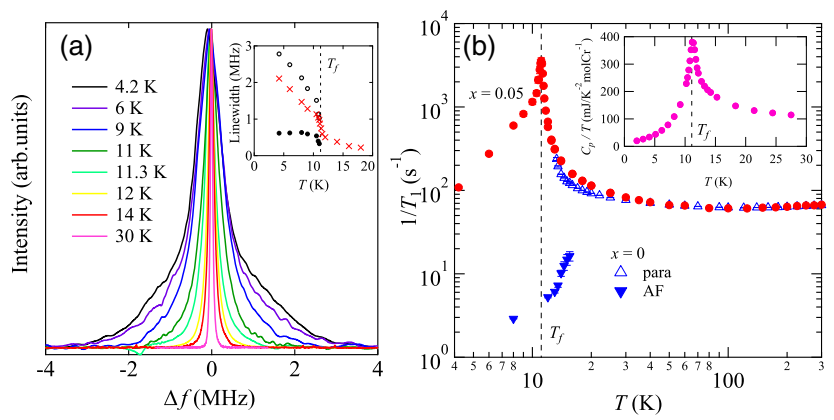

FIG. 1. (a) ${ }^{7} \mathrm{Li}-\mathrm{NMR}$ spectra, normalized by the peak intensity. Here, $\Delta f=0$ corresponds to the center of gravity. The inset shows the temperature dependence of the averaged full-spectral line width (red crosses) and the FWHM of the narrow (closed circles) and broad (open circles) components. (b) The temperature dependence of $1 / T_{1}$ for $x=0.05$ (solid circles), compared with the paramagnetic (open triangles) and AF (solid triangles) components of $x=0$, respectively. The inset shows the temperature dependence of the specific heat divided by temperature $C_{p} / T$ of $x=0.05$. magnetic diffuse scattering was isolated from the remainder by removing the nuclear Bragg features, then subtracting a background to yield zero scattering below $Q=0.4 \AA^{-1}$ [26]. Reverse Monte Carlo (RMC) refinements were performed on the resulting data using the SPINVERT package [28].

The temperature dependence of the ${ }^{7} \mathrm{Li}-\mathrm{NMR}$ spectrum is shown in Fig. 1(a). In the paramagnetic state, it consists of a sharp single line without quadrupole structure, similar to $x=0$. Below $11 \mathrm{~K}$, however, the spectrum shows a marked broadening, indicating the development of a static internal field as a result of spin freezing. The spectra in the lowtemperature phase consist of two components: a relatively narrow line whose width saturates below $9 \mathrm{~K}$, and a broader one that broadens further with decreasing temperature [Fig. 1(a), inset]. The intensity ratio of sharp and broad components of the spectra is estimated to be 1:4.7 at $4.2 \mathrm{~K}[26]$.

Figure 1(b) shows the temperature dependence of $1 / T_{1}$ for both the $x=0.05$ and $x=0$ compositions [29]. The former exhibits a sharp peak in $1 / T_{1}$ at $T_{f}=11.08(5) \mathrm{K}$, indicating critical slowing down associated with a bulk second-order magnetic transition. This transition is also evidenced by a sharp specific heat anomaly at 11.29(3) K [Fig. 1(b), inset]. These behaviors are in contrast with the $x=0$ compound, which shows two first-order magnetic transitions and phase separation [Fig. 1(b)].

Despite observing the clear signatures of a second-order transition in $1 / T_{1}$ and specific heat, both SXRD and ND

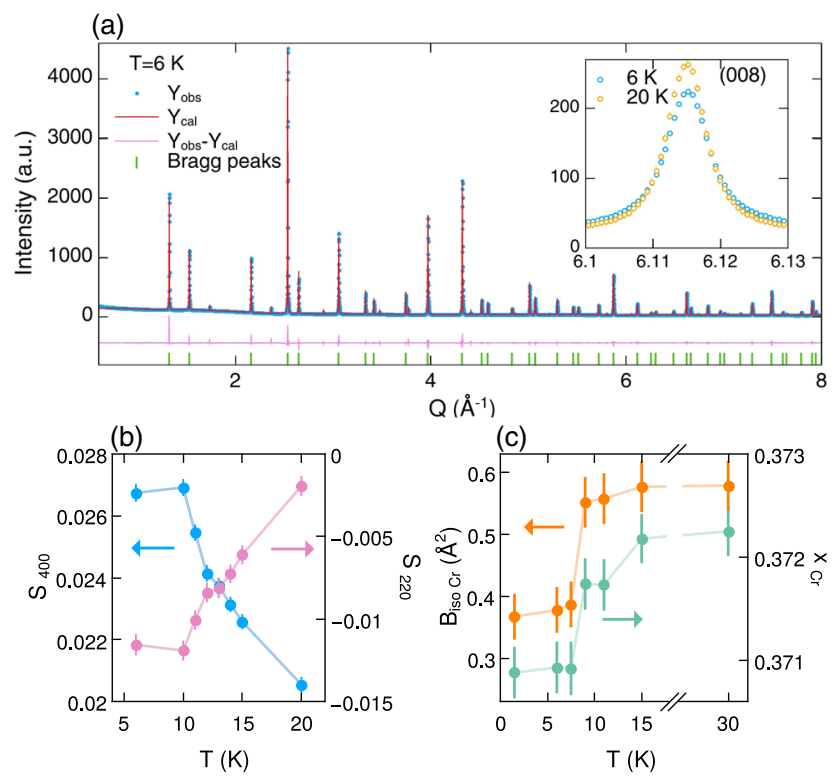

FIG. 2. (a) Synchrotron x-ray pattern at low and high $T$ with its Rietveld refinement $\left(R_{p}=12.2 R_{w p}=15.6 R_{e}=4.05\right)$ and the $T$-dependence of (008) peak (inset). Peaks visible in the measured pattern and not signed with green markers come from a $\sim 1 \% \mathrm{Cr}_{2} \mathrm{O}_{3}$ impurity. Parameters resulting from the structure refinement: temperature dependence of (b) strain parameters $S_{400}$ and $S_{220}$, extracted from SXRD, and (c) $B_{\text {iso }}$ and $\mathrm{Cr}$ fractional position parameter $x$ obtained from ND. 
surprisingly indicate an absence of structural symmetry breaking below $T_{f}$, unlike all other chromate spinels with well-defined phase transitions [Fig. 2(a)]. The only antiferromagnetic order compatible with preserved cubic structural symmetry is the so-called all-in all-out structure, where the spins lie along the local $\langle 111\rangle$ axes. On the other hand, this order implies zero internal field at the ${ }^{7} \mathrm{Li}$ site, in contradiction with the broad experimental NMR spectrum. In addition, no sharp features consistent with all-in all-out order seen in the low-temperature ND data [Fig. 3(a)].

While no diffraction peaks are found to split on crossing $T_{f}$, some broadening of peaks with indices $(h 00)$ and $(h k 0)$ is observed. This implies local tetragonal symmetry, as expected from magnetoelastic coupling within the BBM [8]. To identify the changes in the structure on crossing $T_{f}$, we plot the temperature dependence of the $S_{400}$ and $S_{220}$ strain broadening parameters corresponding to these families of peaks [31], as well as the fractional $\mathrm{Cr}^{3+}$ position and isotropic displacement parameters in Figs. 2(b) and 2(c). All parameters are found to evolve continuously before freezing at $T_{f}$, with the latter doing so in a steplike fashion. This emphasizes the strong magnetoelastic coupling in the system. Furthermore, the freezing of all parameters below $T_{f}$ is consistent with the spin freezing observed in NMR.

Turning to the magnetic diffuse scattering, the data at $30 \mathrm{~K}>T_{f}$ show a broad diffuse feature with a maximum around $Q=1.55 \AA^{-1}$ [Fig. 3(a)]. This is compatible with expectations for the undistorted pyrochlore lattice and implies the presence of Coulomb-like power-law spin-spin correlations [33]. Cooling to $15 \mathrm{~K}$, a weak, but sharp peak is observed at the (110) position, as for $x=0$. Because this appears above $T_{f}$ and seems to be temperature independent on further cooling, it is ascribed to the presence of a small amount of $x=0$ phase in the sample. The only intrinsic changes in the magnetic scattering on crossing the transition are therefore a slight redistribution of scattering towards the positions $Q=0.8 \AA^{-1}$ [near (100)], $1.1 \AA^{-1}$ (110), $1.73 \AA^{-1}$ (210), and $1.87 \AA^{-1}$ (112) [Fig. 3(a)].

To understand the apparent paradox of the presence of a phase transition, on the one hand, and the absence of any peak splittings or (intrinsic) magnetic peaks, on the other, we investigate the changes in the real-space spin-spin correlations via RMC analysis of the magnetic diffuse scattering [34]. At $30 \mathrm{~K}$, the extracted normalized realspace spin-spin correlation function $\left\langle S_{0} \cdot S_{i}\right\rangle / S(S+1)$ indicates antiferromagnetic nearest-neighbor correlations, with $\left\langle S_{0} \cdot S_{1}\right\rangle / S(S+1)=-0.2$ [Fig. 3(b)]. This is consistent with conventional MC simulations of both the undistorted and "breathing" pyrochlore lattices at finite temperature, where the ground states are Coulomb liquids $[9,30]$. However, the correlations do not quite follow the expected form: For example, $\left\langle S_{0} \cdot S_{3}\right\rangle / S(S+1)$, which corresponds to the next-nearest-neighbor distance along $\langle 110\rangle$, is negative rather than positive [9].

Reconstructing the single crystal scattering in the $(h h l)$ [Fig. 3(c)] and (hk0) planes from the RMC spin (a)

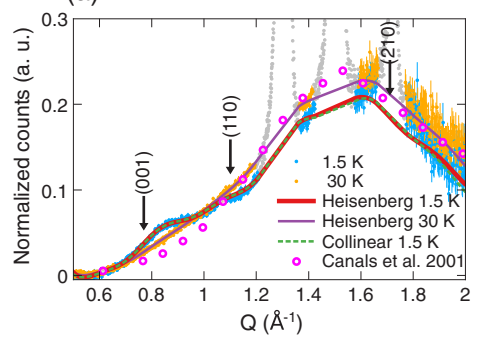

(b)
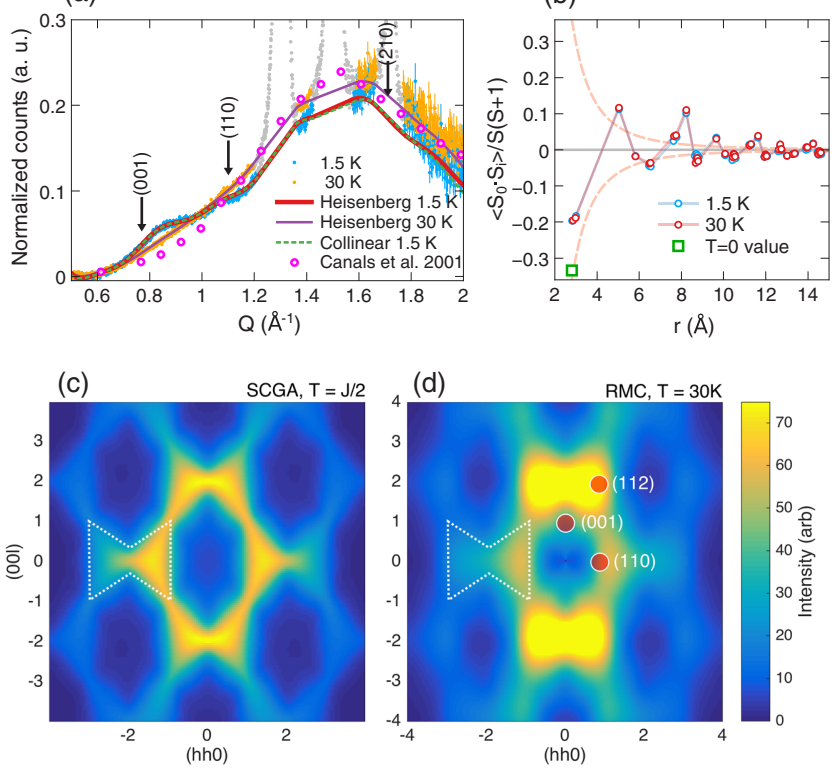

FIG. 3. (a) Diffuse scattering at $1.5 \mathrm{~K}$ and $30 \mathrm{~K}$ with RMC fits using free (Heisenberg) and collinear spins parallel to (001). (b) Normalized real-space spin-spin correlation functions $\left\langle S_{0} \cdot S_{i}\right\rangle / S(S+1)$ versus radial distance $r$ for both temperatures. The dashed lines indicate the expected envelope for the Coulomblike phase $\propto 1 / r^{3}$, and the green square is the expectation for $\left\langle S_{0} \cdot S_{1}\right\rangle / S(S+1)$ as $T \rightarrow 0$. (d) Reconstructed single crystal scattering from the 30-K Heisenberg fit compared with (c) bowtie scattering (highlighted by the white dashed polygon) calculated for the breathing pyrochlore antiferromagnet within the self-consistent Gaussian approximation (SCGA) for $B_{f}=0.6$ and $T / J=0.5[30]$.

configurations [28] provides some clues as to why this is the case: Intensity is observed at positions consistent with the propagation vector $\mathbf{k}=(001)$, which manifests in our experiments as scattering around the $Q$ listed previously. The scattering maps also allow us to identify "bow-tie" features characteristic of the underlying Coulomb liquid state [30] [Figs. 3(c) and 3(d)]. The overall picture of $\mathbf{k}=(001)$ short-range order superimposed on bow-tie features remains unchanged to $1.5 \mathrm{~K}$, although the former grows slightly in intensity below $T_{f}$. This is also true of $\left\langle S_{0} \cdot S_{i}\right\rangle / S(S+1)$, which is nearly indistinguishable between the high- and low-temperature data sets [Fig. 3(b)], emphasising that the order parameter of the transition cannot be dipolar.

Classical MC simulations of the BBM with Gaussian bond disorder $\Delta$ (here related to the substitution $x$ ) indicate a quadrupolar nematic transition to a collinear state with persistent Coulomb-like correlations for $b<0, \mathcal{P}=0$, and small $\Delta$ [23]. As $\Delta(x)$ is increased, the nematic transition becomes concurrent with spin freezing. In the present compound, because of (i) the lack of magnetic Bragg peaks at $T<T_{f}$, despite a clear phase transition; (ii) the tendency towards collinear spin arrangements in $x=0,1$, implying $b<0$ for all $x$; (iii) the local tetragonal symmetry, 
consistent with such spin arrangements; and (iv) the spin freezing observed in NMR, we speculatively assign the phase transition to concurrent nematic order and spin freezing. To test this assignment, we perform further RMC simulations on the $1.5-\mathrm{K}$ data with the spins constrained to lie along any of three high-symmetry directions in the cell $[(001),(110)$, and (111)]-i.e., collinear spins [35]. Several directions are modeled due to the cubic symmetry of the system; while all should reproduce the experimental scattering, the corresponding spin configurations generally differ. With collinear spins, the data are modeled nearly as well as the Heisenberg case $\left(\chi_{\text {Ising }}^{2} / \chi_{\text {Heis }}^{2} \sim 1.05\right)$ for all spin directions.

To verify that this assumption is consistent with our NMR results, we simulate the NMR spectrum corresponding to the collinear RMC spin configurations [26]. The main component of the simulated spectrum, ascribed to the broad part of the magnetic diffuse scattering (and hence the bow-tie features), is triangular and reproduces the shape of the broad component in the 4.2-K NMR spectrum. On the other hand, the sharp component, corresponding to the $\mathbf{k}=(001)$ short-range order is slightly underestimated [26]. Scaling the width of the triangular component to the NMR data, the $\mathrm{Cr}^{3+}$ moment size is estimated to be $g S=1.3 \mu_{B}$ at $4.2 \mathrm{~K}$. Although this quantity is model dependent, the reduced moment could indicate the presence of fast quantum or thermal fluctuations in the ground state.

The RMC spin configurations may be further analyzed by examining the collinear spin arrangements on individual tetrahedra: For all directions of the easy axis, arrangements with two spins up and two down (uudd) are favored over other configurations. However, the ratio of $u u d d$ to threeup one-down (uuud, or vice versa, $u d d d$ ) arrangements is typically in excess of 8 for the (001) axis, versus $2-4$ for the other directions. Assuming that all nearest-neighbor interactions in the system are antiferromagnetic, this solution is clearly the most energetically favorable, and we will therefore focus on it in the subsequent analysis.

Following Tchernyshyov et al. [8], each uudd tetrahedron may be given a color according to the arrangement of the two ferromagnetic and four antiferromagnetic bonds and, correspondingly, the direction of the local tetragonal distortion. Ferromagnetic (long) bonds along $\langle 110\rangle$ correspond to blue $(B),\langle 101\rangle$ green $(G)$, and $\langle 011\rangle$ red $(R)$. In the case of the $x=0$ and $x=1$ compounds, the magnetic orders may be described as $R G$ and $B G$ color arrangements on the diamond lattice formed by the tetrahedra, with the spins directed along the $c$ axis of the tetragonal cell. In the present case, the RMC spin configurations exhibit majority of $B$ tetrahedra. As such the $\mathbf{k}=(001)$ scattering may be associated with domains containing combinations of $B R$ and $B G$. Interestingly, this means that the ground state of the present material lies closer to the $x=1$ ordered structure, despite its chemical proximity to the $x=0$
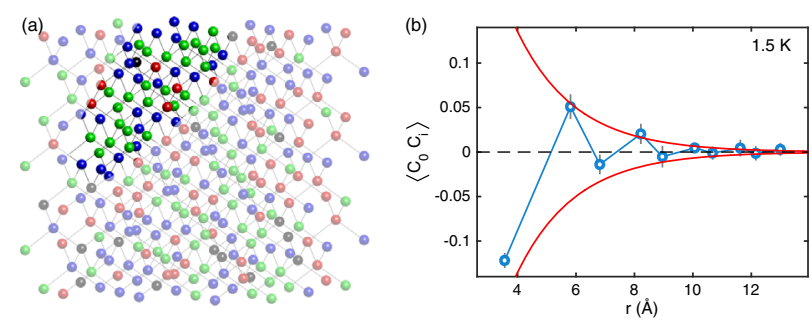

FIG. 4. (a) Real-space plot of a color RMC configuration at $1.5 \mathrm{~K}(2 \times 2 \times 2$ unit cells $)$. The black spheres indicate uuud tetrahedra (or vice versa), and the highlighted area shows a region with predominantly blue-green correlations. (b) Radial dependence of color-color correlations $\left\langle C_{0} C_{i}\right\rangle$ at $1.5 \mathrm{~K}$. The red lines correspond to the expected envelope for two-color correlations decaying exponentially with the correlation length $\xi_{c}=2 \AA$.

compound. The $B R / B G$ short-range order is furthermore consistent with the negative $\left\langle S_{0} \cdot S_{3}\right\rangle / S(S+1)$ found in the Heisenberg fits, as well as the broadening of the structural diffraction peaks.

To quantify the spatial extent of the opposite color correlations, we compute a color correlation function $\left\langle C_{0} C_{i}\right\rangle$, defined such that $\left\langle C_{0} C_{i}\right\rangle=1$ for the same and $\left\langle C_{0} C_{i}\right\rangle=-1$ for different colors between tetrahedra. We thus find $\left\langle C_{0} C_{1}\right\rangle=-0.14$ and opposite-color short-range order, with an exponential radial decay characterized by the correlation length $\xi_{c}=2 \AA$ [Fig. 4(b)]. While this is short, $B G$ and $B R$ domains as large as $20 \AA$ can be identified by visual inspection of the color configurations [Fig. 4(a)]. The scattering around (001) $\left(0.8 \AA^{-1}\right)$ —which would vanish in the case of perfect color order-can thus be understood as resulting from disorder between the minority colors $(R$ and $G)$ in the overall two-color pattern. In this sense, the low-temperature state shows some commonalities with the partially ordered phase predicted from MC simulations of the pyrochlore lattice with further neighbor couplings [11] and a three-state Potts model on the diamond lattice [36]. The partially ordered phase has a single color (here $B$ ) on all up-pointing tetrahedra (diamond lattice nodes), and a distribution of other colors on downpointing tetrahedra (here $G$ and $R$ ).

There are, however, some uncertainties surrounding the above interpretation. Most importantly, existing MC simulations only consider the undistorted pyrochlore lattice, and it is not known how the breathing distortion changes the phase diagram. Indeed, in the undistorted case, the transition is expected to be first order for small $x$, only becoming second order deep inside the spin glass regime, where the nematic transition coincides with spin freezing. This contradicts our observation of a second-order transition and spin freezing at small $x$, as well as the appearance of a conventional spin glass phase at larger $x$ [21]. On the other hand, the nematic frozen state is expected to be exceptionally robust towards the magnetic field, much like the present low-temperature phase [26]—similar behavior 
is also observed below $T_{f}$ in pure $\mathrm{Y}_{2} \mathrm{Mo}_{2} \mathrm{O}_{7}$, where no clear nematic transition is observed. Finally, it is not evident why the nematic transition occurs at all, given the presence of further neighbor couplings.

To conclude, we have shown that $\mathrm{LiGa}_{0.95} \mathrm{In}_{0.05} \mathrm{Cr}_{4} \mathrm{O}_{8}$ undergoes a single second-order transition at $T_{f}=11 \mathrm{~K}$. This transition corresponds neither to magnetic long-range order nor to a structural symmetry breaking, but is rather ascribed to nematic (collinear) spin freezing. Upon cooling, correlations corresponding to the propagation vector $\mathbf{k}=(001)$ are enhanced. Assuming that the spins lie along (001), these correspond to short-range order between collinear spin configurations on the tetrahedra. The lowtemperature state thus shares features with both the nematic and partially ordered states anticipated for the pyrochlore Heisenberg model with perturbations.

We gratefully acknowledge T. Fennell for his careful reading of and useful comments on the manuscript. We also thank N. Shannon and O. Benton for sharing their SCGA data, and S. Hayashida and J. A. M. Paddison for useful discussions. This work was supported by JSPS KAKENHI (Grants No. 25287083, No. 16J01077, and No. 17H02918). Y. T. was supported by the JSPS through the Program for Leading Graduate Schools (MERIT).

*wawrzynczak@ill.eu

†you@issp.u-tokyo.ac.jp

¥goran.nilsen@stfc.ac.uk

[1] V. Fritsch, J. Hemberger, N. Büttgen, E.-W. Scheidt, H.-A. K. von Nidda, A. Loidl, and V. Tsurkan, Phys. Rev. Lett. 92, 116401 (2004).

[2] I. Kézsmárki, S. Bordács, P. Milde, E. Neuber, L. M. Eng, J. S. White, H. M. Rønnow, C. D. Dewhurst, M. Mochizuki, K. Yanai et al., Nat. Mater. 14, 1116 (2015).

[3] S.-H. Lee, C. Broholm, T. H. Kim, W. Ratcliff, and S.-W. Cheong, Phys. Rev. Lett. 84, 3718 (2000).

[4] S. Ji, S.-H. Lee, C. Broholm, T. Y. Koo, W. Ratcliff, S.-W. Cheong, and P. Zschack, Phys. Rev. Lett. 103, 037201 (2009).

[5] S. Bordács, D. Varjas, I. Kézsmárki, G. Mihály, L. Baldassarre, A. Abouelsayed, C. A. Kuntscher, K. Ohgushi, and Y. Tokura, Phys. Rev. Lett. 103, 077205 (2009).

[6] G. J. Nilsen, Y. Okamoto, T. Masuda, J. Rodriguez-Carvajal, H. Mutka, T. Hansen, and Z. Hiroi, Phys. Rev. B 91, 174435 (2015).

[7] J.-H. Chung, M. Matsuda, S.-H. Lee, K. Kakurai, H. Ueda, T. J. Sato, H. Takagi, K.-P. Hong, and S. Park, Phys. Rev. Lett. 95, 247204 (2005).

[8] O. Tchernyshyov, R. Moessner, and S. L. Sondhi, Phys. Rev. B 66, 064403 (2002).

[9] N. Shannon, K. Penc, and Y. Motome, Phys. Rev. B 81, 184409 (2010).

[10] G.-W. Chern, C. J. Fennie, and O. Tchernyshyov, Phys. Rev. B 74, 060405 (2006).

[11] G.-W. Chern, R. Moessner, and O. Tchernyshyov, Phys. Rev. B 78, 144418 (2008).
[12] Y. Okamoto, G. J. Nilsen, J. P. Attfield, and Z. Hiroi, Phys. Rev. Lett. 110, 097203 (2013).

[13] M. C. Kemei, S. L. Moffitt, L. E. Darago, R. Seshadri, M. R. Suchomel, D. P. Shoemaker, K. Page, and J. Siewenie, Phys. Rev. B 89, 174410 (2014).

[14] M. C. Kemei, P. T. Barton, S. L. Moffitt, M. W. Gaultois, J. A. Kurzman, R. Seshadri, M. R. Suchomel, and Y.-II Kim, J. Phys. Condens. Matter 25, 326001 (2013).

[15] K. Tomiyasu, H. Suzuki, M. Toki, S. Itoh, M. Matsuura, N. Aso, and K. Yamada, Phys. Rev. Lett. 101, 177401 (2008).

[16] We believe this to be the magnetic space group $C_{A} 222_{1}$, belonging to the $m M_{5}$ irreducible representation [MillerLove notation, parent space group $\operatorname{Im} 2, \mathbf{k}=(001)]$ [17], rather than $P_{I} m 2\left(m M_{1}\right)$, as proposed in Ref. [18].

[17] R. Wawrzyńczak, G. J. Nilsen, Y. Okamoto, and Z. Hiroi, ILL Experimental Report, Proposal No. 5-31-2396, available at http://club.ill.fr/cv/servlet/ReportFind.

[18] R. Saha, F. Fauth, M. Avdeev, P. Kayser, B. J. Kennedy, and A. Sundaresan, Phys. Rev. B 94, 064420 (2016).

[19] R. Wawrzyńczak, G. J. Nilsen, N. Casati, Y. Okamoto, and Z. Hiroi, PSI Experimental Report: SLS Proposal No. 20150562, available at http://duo.psi.ch.

[20] Y. Tanaka, M. Yoshida, M. Takigawa, Y. Okamoto, and Z. Hiroi, Phys. Rev. Lett. 113, 227204 (2014).

[21] Y. Okamoto, G. J. Nilsen, T. Nakazano, and Z. Hiroi, J. Phys. Soc. Jpn. 84, 043707 (2015).

[22] W. Ratcliff, S.-H. Lee, C. Broholm, S.-W. Cheong, and Q. Huang, Phys. Rev. B 65, 220406 (2002).

[23] H. Shinaoka, Y. Tomita, and Y. Motome, Phys. Rev. B 90, 165119 (2014).

[24] H. J. Silverstein, K. Fritsch, F. Flicker, A. M. Hallas, J. S. Gardner, Y. Qiu, G. Ehlers, A. T. Savici, Z. Yamani, K. A. Ross et al., Phys. Rev. B 89, 054433 (2014).

[25] J. S. Gardner, B. D. Gaulin, S.-H. Lee, C. Broholm, N. P. Raju, and J. E. Greedan, Phys. Rev. Lett. 83, 211 (1999).

[26] See Supplemental Material at http://link.aps.org/ supplemental/10.1103/PhysRevLett.119.087201 for additional information on the NMR measurements the neutron and synchrotron powder diffraction refinements and reverse Monte-Carlo simulations.

[27] J. Rodriguez-Carvajal, Physica B (Amsterdam) 192, 55 (1993).

[28] J. A. M. Paddison, J. R. Stewart, and A. L. Goodwin, J. Phys. Condens. Matter 25, 454220 (2013).

[29] The temperature dependence of $\beta$ is shown in Ref. [26].

[30] O. Benton and N. Shannon, J. Phys. Soc. Jpn. 84, 104710 (2015).

[31] $S_{h k l}$ is proportional to the variance of the interplanar distance [32].

[32] P. W. Stephens, J. Appl. Crystallogr. 32, 281 (1999).

[33] S. V. Isakov, K. Gregor, R. Moessner, and S. L. Sondhi, Phys. Rev. Lett. 93, 167204 (2004).

[34] J. A. M. Paddison, J. R. Stewart, and A. L. Goodwin, J. Phys. Condens. Matter 25, 454220 (2013).

[35] We note that the assumption of collinear spins cannot be justified on the basis of a finite quadrupolar order parameter from the high-temperature Heisenberg fits, and that the Ising fits do not converge at high temperature.

[36] S. Lapinskas and A. Rosengren, Phys. Rev. Lett. 81, 1302 (1998). 